\title{
Competing priorities and the NHS
}

\author{
José G Merino US clinical research editor
}

The BMJ

Since the NHS was founded 70 years ago, the proportion of GDP that the UK spends on healthcare has doubled to around $10 \%$, a level matching that of other European countries. As Anita Charlesworth and Karen Bloor argue in their analysis (doi:10.1136/bmj.k2373), the decision on how much to spend on healthcare involves tradeoffs between competing priorities and sources of revenue and is, at its core, political.

Over the next five years NHS England will receive an average annual funding increase of $3.4 \%$, and areas such as public health, social care, training, and capital investment will be considered separately in the budget (doi:10.1136/bmj.k2682). This extra funding is intended to strengthen the NHS in the long term and help tackle some of the challenges it faces, including access, waiting times, and staffing levels. The new funds will reportedly come from the "Brexit dividend" and taxes. Charlesworth and Bloor argue that general taxation is the most efficient and equitable way to generate funds for the NHS. David Oliver worries that calling the new money "Brexit dividend" is political spin (doi:10.1136/bmj.k2645).

Extra funding by itself will not eliminate all the NHS's challenges. Staffing shortages, for example, were compounded by a cap on tier 2 skilled worker visas for doctors and nurses. Last month The BMJ launched its Scrap the Cap campaign (bmj. com/scrap-the-cap), and last week the Home Office announced that it will remove these visa restrictions (doi:10.1136/bmj. k2648). Further measures that reduce waste, prevent staff burnout, and improve clinical outcomes will help preserve the public's trust in the NHS. And Ben Page of Ipsos MORI argues that a change in the public's attitude to health and a shift from illness to wellness are necessary to avoid future crises in the NHS (doi:10.1136/bmj.k2663).

This week two articles tackle challenging imaging questions. An umbrella review in our research section looks at the prevalence and implications (in terms of malignant lesions) of incidental findings (incidentalomas) in imaging studies (doi:10. 1136/bmj.k2387). The authors found large variability across different imaging techniques and body parts. An accompanying editorial highlights the importance of discussing the issue of incidentalomas with patients when imaging studies are contemplated (doi:10.1136/bmj.k2611).

An education article looks at the indications, risks, and benefits of different abdominal imaging modalities for women in the third trimester of pregnancy (doi:10.1136/bmj.k2511): magnetic imaging and ultrasonography are the preferred imaging methods; computed tomography should be used with caution and always after discussion with patients of radiation risks. 